\title{
REVIEW
}

\section{The Role of Computed Tomographic Colonography in Colorectal Cancer Screening}

\author{
André R. Maddison', Geoff Williams MD² \\ ${ }^{1}$ Class of 2014, Faculty of Medicine, Dalhousie University \\ ${ }^{2}$ Division of Gastroenterology, Department of Medicine, Dalhousie University
}

\begin{abstract}
Objective: We conducted a literature review to identify the current state of knowledge regarding the optimal clinical use of computed tomographic colonography (CTC) in Canada, based on accuracy, patient safety, and costeffectiveness.

Methods: Articles were retrieved from PubMed and the Cochrane Library. Retrieved studies were included based on relevance and appropriateness as determined by reviewing titles and abstracts. Studies were excluded if they were duplicated, grey literature, or non-peer-reviewed. Of the studies remaining after exclusions, reference lists were scanned to obtain further relevant articles.

Results: The literature reports comparable accuracy for detecting cancers and large polyps, yet CTC is less sensitive than colonoscopy for detecting small polyps. Most would agree that CTC is safer than colonoscopy, yet it is not without risk or adverse events. Lastly, although the true costs of CTC vs. colonoscopy are complex, the literature consistently demonstrates that CRC screening with CTC is less cost-effective than screening with colonoscopy.

Conclusion: Unless there are modifications to CTC that improve cost-effectiveness and/or accuracy, the future of CRC screening in Canada will remain reliant on colonoscopy. CTC is beneficial as an alternative to colonoscopy, but should remain available for selected indications. CTC has value, however, it has fallen short of initial expectations.
\end{abstract}

When computed tomographic colonography (CTC) was first described in 1994, there was optimism among the medical community involved in colorectal cancer $(\mathrm{CRC})$ screening that CTC might be a safer, less expensive, and less invasive alternative to colonoscopy. Moreover, it was hoped that CTC would reduce the demand for colonoscopy, which could be reserved for therapeutic use. However, 18 years later, colonoscopy continues to play a fundamental role in CRC screening, while CTC is used infrequently. ${ }^{1,2}$ As more Canadian provinces roll out population-wide CRC screening programs centered on Fecal Occult Blood Testing (FOBT), Fecal Immunochemical Testing (FIT) and colonoscopy, where does this leave CTC?

CTC is an abdominal computed tomography scan of an insufflated colon and rectum. CTC is done with the patient in prone and supine positions. Similar to colonoscopy, patients are required to undergo bowel preparation. Additionally, patients may be given oral barium fluid to enhance contrast, yet intravenous contrast is not required. The colon and rectum are inflated with carbon dioxide via a rectal catheter. ${ }^{3}$ The inflation of the colon and the bowel preparation are done in order to improve the clarity and accuracy of imaging. CTC is conducted by a lab technician and analyzed and interpreted by a radiologist and/or gastroenterologist. The CT images provide a view in any plane, and can be assembled to become a virtual colonoscopy. The virtual colonoscopy enables the radiologist or gastroenterologist to view the colon as is if through a colonoscopy probe. ${ }^{3}$

Colonoscopy is a widely used endoscopic examination of the rectum and large intestine, which is highly sensitive and specific for identifying pre-cancerous polyps. ${ }^{4}$ Colonoscopy has the advantage of being both diagnostic and therapeutic, as detected polyps can be excised during the procedure. However, it is invasive, operator dependent, and carries risks that are not seen with CTC. Each procedure carries a 1/500 - 1/1,000 risk of major complication, such as bowel perforation or bleeding. ${ }^{5}$ Colonoscopy is a procedure in which the accuracy of detection and safety are based on the skills and experience of the physician, thereby introducing variation in detection and treatment. ${ }^{5}$ Furthermore, the invasiveness of the procedure and discomfort caused by the bowel preparation may contribute to sub-optimal patient adherence to CRC screening. ${ }^{6}$ Lastly, as $98 \%$ of colonoscopies are done under conscious sedation, 
patients are considered legally impaired for 24 hours after the procedure, and therefore, are absent from work or school during this time. ${ }^{7}$

$\mathrm{CRC}$ is the third most common cancer in Canada among men and women and there is strong evidence to support population-wide screening. ${ }^{1,8}$ This article reviews the optimal clinical use of CTC in Canada, based on test accuracy, patient safety, and cost-effectiveness.

\section{Methods}

The literature review was conducted using PubMed and Cochrane Library. The literature search applied MESH terms in PubMed and keywords in Cochrane (Figure 1). The search strategy was as follows: 'CT colonography' and 'cost-effectivess' or 'safety' or 'accuracy' or 'colonoscopy', which retrieved 1,373 studies. Literature searches were not restricted by year of publication or journal. Studies were assessed based on relevance and appropriateness by reviewing titles and abstracts. Additionally, studies were excluded if they were duplicated, not English language, grey literature, or non-peer-reviewed. Of the studies remaining after exclusions, reference lists were scanned to obtain further relevant articles. In total, 27 articles were considered relevant and appropriate based on their contribution to the literature and were included in the review.

\section{Results}

The initial studies assessing the accuracy of CTC reported promising results, but had significant variability and methodological shortcomings. Fenlon et al. published the first study evaluating CTC in 1999. ${ }^{9}$ The authors assessed the ability of CTC to detect polyps in 100 patients being screened for CRC, using colonoscopy as the gold standard. They reported a sensitivity of $96 \%$ and $94 \%$ for polyps $\geq 1 \mathrm{~cm}$ and 6-9 mm, respectively. ${ }^{9}$ Similarly, Yee et al. evaluated the accuracy of CTC on 300 patients, published in $2001 .{ }^{10}$ They identified sensitivities of $94 \%$ and $82 \%$ for adenomas of $\geq 1 \mathrm{~cm}$ and $5-9 \mathrm{~mm}$, respectively. ${ }^{10}$ The evidence for CTC was further strengthened in a 2003 study by Pickhardt et al. published in the New England Journal of Medicine. ${ }^{11}$ Among 1,233 asymptomatic adults, they reported sensitivities of $88.7 \%$, 93.9\%, and $93.8 \%$ for detecting polyps of $\geq 6 \mathrm{~mm}, \geq 8 \mathrm{~mm}$, and $\geq 10 \mathrm{~mm}$, respectively using CTC. Furthermore, they reported that the sensitivity of detecting polyps using CTC surpassed that of colonoscopy for polyps $\geq 8 \mathrm{~mm}$ and $\geq 10 \mathrm{~mm} .{ }^{11}$ At that point in time, CTC was gaining support within research and clinical communities.

In 2005, a meta-analysis of 33 studies was conducted in order to synthesize the evidence to date. ${ }^{12}$ The authors concluded that CTC was highly accurate in detecting polyps $\geq 6 \mathrm{~mm}$, but had significantly lower sensitivity for smaller polyps. They reported a pooled sensitivity for polyps $<6 \mathrm{~mm}$ of $48 \%$. Another meta-analysis, by Halligan et al, revealed further concerns. ${ }^{13}$ They identified that among the 24 studies included, the sensitivity for detecting polyps of all sizes ranged from $45 \%$ to $97 \%$; indicating significant variability between studies. Furthermore, Halligan et al. suggested that

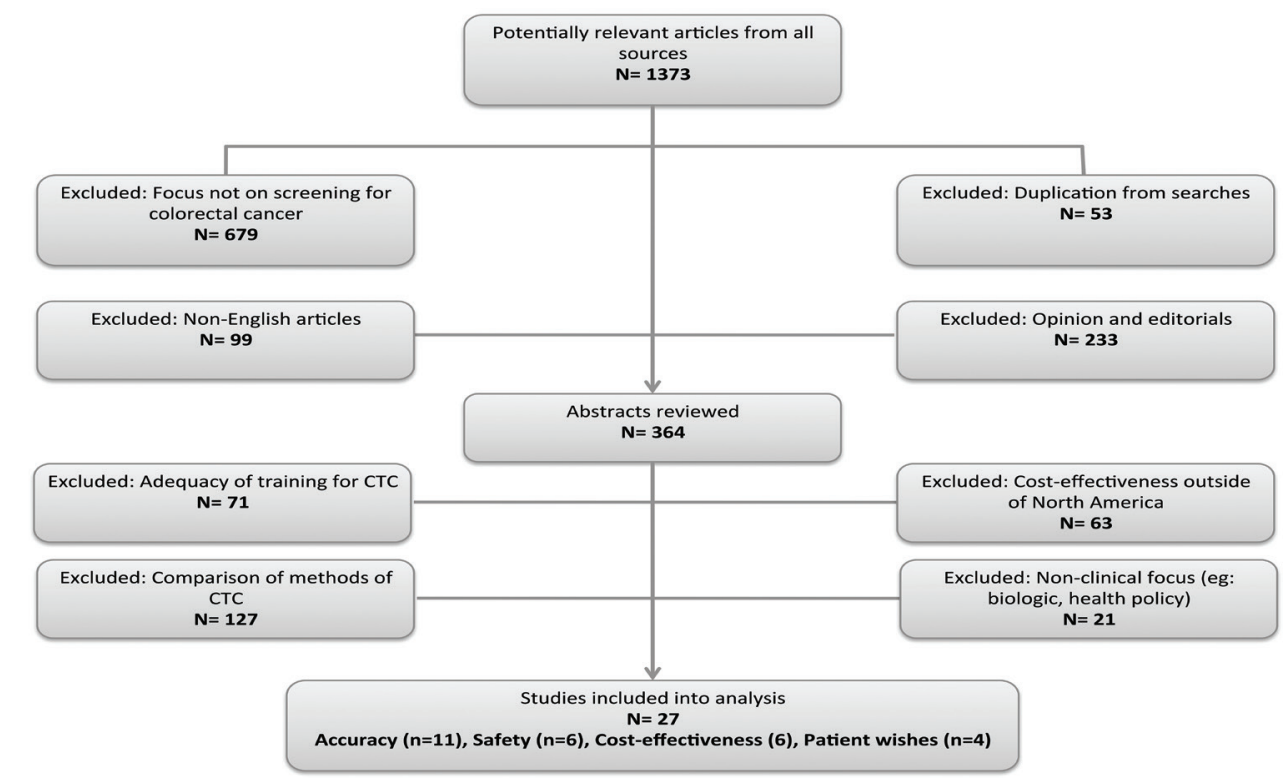

Figure 1. Study selection process. 
several of the studies were conducted on a subset of patients with a greater risk of abnormal findings, thus increasing the sensitivity, compared to the general population. ${ }^{13}$

Since 2005, several high quality meta-analyses and systematic reviews have been published, which have provided further evidence to the growing body of literature. The literature consistently demonstrates that CTC is accurate for detecting colorectal cancers and polyps $>10 \mathrm{~mm}$, but is questionable for smaller polyps. Three meta-analyses demonstrated CTC sensitivities above $80 \%$ for polyps $>9 \mathrm{~mm}$, however, each reported decreases in CTC sensitivity as polyp size diminished. ${ }^{14-16}$ The accuracy of CTC was further analyzed by Walleser et al. who conducted a systematic review focusing on CTC detection of polyps after a positive screening for fecal occult blood. ${ }^{17}$ Based on 5 studies, they reported a pooled sensitivity for detecting all polyps of $63 \%$ for CTC and $95 \%$ for colonoscopy. ${ }^{17}$

The potential complications of colonoscopy are well documented in the literature, and include risk of perforating the bowel, bleeding, infection, as well as risks associated with sedation and bowel preparation. The risk of bowel perforation has been extensively assessed and is commonly reported as between 0.09\% and $0.3 \%$, while risk of bleeding without perforation is between $0.1 \%$ and $0.6 \%{ }^{18}$ Complications due to sedation are commonly cardio-pulmonary in nature, including transient hypoxia, respiratory distress, vasovagal reactions, and arrhythmias. ${ }^{18}$ The principle concern of bowel preparation is the risk of acute nephropathy or deterioration of chronic kidney disease, both of which are rare and typically preventable. ${ }^{18}$ Although CTC is considered safer than colonoscopy, it is not without risk. Each CTC involves radiation exposure, which increases an individual's risk of future malignancy. ${ }^{7}$ Although radiation exposure varies based on the model of CT scanner, on average, CTC has been estimated to carry a $0.14 \%$ absolute lifetime risk of cancer in a 50 year-old individual. ${ }^{19} \mathrm{CTC}$ also carries a risk of perforation caused by insufflation of the bowel. However, the risk of perforation has been reported as $0.009-0.05 \%$, significantly lower than colonoscopy. ${ }^{20}$ Lastly, as CTC also requires individuals to undergo bowel preparation, the risk of renal impairment is comparable to colonoscopy. ${ }^{7}$

The literature consistently demonstrates that CRC screening with CTC is less cost-effective than screening with colonoscopy. Cost-effectiveness compares the effectiveness of a test (i.e., sensitivity and specificity) with the cost of the test or program. A 2011 literature review identified 6 studies from around the world that compared cost-effectiveness of CTC and colonoscopy. In 5 of 6 studies, the conclusion was that screening with CTC was less cost-effective than with colonoscopy. ${ }^{21}$ The only study that did not report CTC as less cost-effective than colonoscopy did not consider the cost of follow-up colonoscopies for polyps $\leq 5 \mathrm{~mm}$, which will be required in $15-25 \%$ of patients. ${ }^{22,23}$ Heitman et al. compared screening by CTC and screening by colonoscopy from a Canadian perspective. ${ }^{25}$ They conducted a decision analysis to assess cost-effectiveness among averagerisk individuals greater than 50 years old. Heitman and colleagues calculated direct costs of CTC and colonoscopy (i.e., physician and nurse costs, medical and surgical supplies, medications, instrument sterilization, and overhead), as well as the cost of missed CRC diagnoses and treatment of possible complications. They concluded that screening with colonoscopy was 2.27 million dollars less per 100,000 individuals screened, compared to CTC. ${ }^{24}$ A further study by Heitman et al. evaluated the nonmedical costs associated with CTC vs. colonoscopy, using a crosssectional survey of patients in Alberta. ${ }^{25}$ Nonmedical costs, also known as indirect costs, included travel costs and time costs (i.e., missed wages) for the patient. They reported nonmedical costs of $\$ 109.30$ for CTC, compared to $\$ 308$ for optical colonoscopy. ${ }^{25}$ The higher nonmedical costs for colonoscopy are due primarily to increased time away from work, compared to CTC. The authors cautioned however, that the nonmedical cost of CTC does not include the cost of follow-up colonoscopy. ${ }^{25}$

Colonoscopy has an intrinsic advantage over CTC, the ability to biopsy and excise detected polyps. Although not easily quantified, the benefit of the therapeutic component of colonoscopy is not negligible. A 2010 pilot project $(n=2005)$ from Vancouver, British Columbia, reported that $25.7 \%$ of patient who received CTC, required follow-up colonoscopy. ${ }^{22}$ The most common indications for follow-up colonoscopy included suspected colonic neoplasm and inadequate view with CTC. ${ }^{22}$ When follow-up colonoscopy is indicated, most centers attempt to fit it in the same day as CTC in order to avoid a second bowel preparation, but this is not always possible. Not only are there financial costs accrued by the health system and patient, but there is also significant stress on the patient due to the "positive" test.

CTC does not image the colon exclusively, and thus has the potential to detect anatomic abnormalities and pathologies of other abdominal, pelvic, and thoracic organs. ${ }^{3}$ Incidental extra-colonic findings 
(ECFs) were initially thought to be a benefit of CTC, as it enabled clinicians to detect possible extra-colonic malignancies, abdominal aortic aneurysms, cirrhosis, and hydronephrosis. ${ }^{26}$ However, the tune has changed for incidental ECFs, which are now considered a burden and potential danger of CTC. ${ }^{26}$ Incidental ECFs are common, infrequently detect a significant pathology, and result in significant patient burden and health care costs. ${ }^{26}$ A literature review of 18 studies reported that of a cumulative 3,280 patient who underwent CTC, 1,763 incidental findings were detected, of which only $3.7 \%$ were cancers or aortic abdominal aneurysms. ${ }^{27}$ In addition to the emotional burden on patients, it has also been estimated that incidental ECFs add \$297 (US) per patient to the overall cost of CTC. ${ }^{27}$ Therefore, although ECFs are a potential benefit of CTC, the financial cost, human resource burden, and patient impact cannot be overlooked.

Patient perspectives of medical tests influence patient adherence, and therefore the effectiveness of a screening program. On average, patients report being satisfied with colonoscopy and 73 to $100 \%$ state they are willing to return. ${ }^{28}$ Five studies comparing patient preferences of CTC vs. colonoscopy demonstrate inconsistent findings. Three of the studies reported that the majority of patients preferred CTC, while the remaining 2 studies reported that the majority of patients preferred colonoscopy. ${ }^{28}$

\section{Discussion}

When first described, it was anticipated that CTC would replace colonoscopy as a safer and less expensive option for CRC screening. It is currently a consensus that CTC will not simply replace colonoscopy. The literature reports comparable accuracy for detecting cancers and large polyps, yet CTC is less sensitive than colonoscopy for detecting small polyps. Most would agree that CTC is safer than colonoscopy, yet it is not without risk or adverse events. Lastly, although the true costs of CTC vs. colonoscopy are complex, most experts agree that colonoscopy is more cost-effective than CTC. ${ }^{7}$

The Canadian Association of Gastroenterology (CAG) and Canadian Digestive Health Foundation recommend biennial FOBT or FIT for population-wide screening of CRC. ${ }^{2}$ They put forth that colonoscopy be restricted to screening of higher risk individuals and follow-up for abnormal FOBT or FIT; and endorse the use of CTC for "selected indications"., The CAG published a position statement in 2010, which revised the most recent Canadian guidelines, and introduced potential indications for CTC. ${ }^{7}$ Approximately 13\% of colonoscopies are not completed, due primarily to colonic obstructions (e.g. tumour or diverticulosis) or a tortuous colon. ${ }^{29}$ Patients with an incomplete colonoscopy may benefit from CTC to visualize areas of the colon not seen by endoscopy. CTC may also be done on a case-by-case basis for patients with severe cardiopulmonary co-morbidities to avoid sedation, as well as those who are anticoagulated in order to minimize the complication from perforation. ${ }^{7}$ However, no provincial CRC screening programs currently include or recommend CTC. ${ }^{30}$ Due to the lack of specific protocol, CTC use in Canada varies based on local preferences, human resource availability, and access to equipment.

Unless there are modifications to CTC that improve cost-effectiveness and/or accuracy, the future of CRC screening in Canada will remain reliant on colonoscopy. Simply stated, no aspect of CTC merits an overhaul of provincial screening programs. CTC is beneficial as an alternative to colonoscopy, but should remain available for the specific scenarios described above. CTC has value, however, it has fallen short of initial expectations.

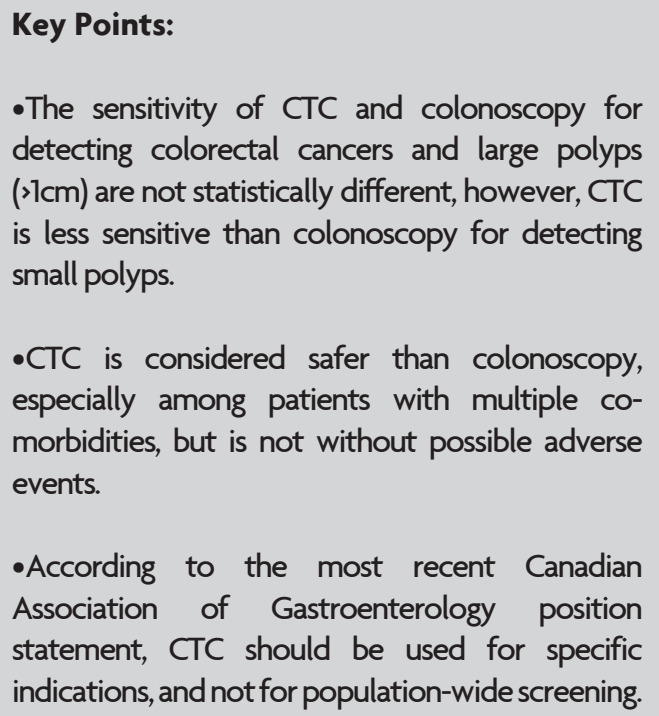
$(>\mathrm{cm})$ are not statistically different, however, CTC is less sensitive than colonoscopy for detecting small polyps.

-CTC is considered safer than colonoscopy, especially among patients with multiple comorbidities, but is not without possible adverse events.

-According to the most recent Canadian Association of Gastroenterology position statement, CTC should be used for specific indications, and not for population-wide screening.

\section{References}

1. Leddin D, Hunt R, Champion M, Cockeram A, Flook N, Gould $\mathrm{M}$, et al. Canadian Association of Gastroenterology and the Canadian Digestive Health Foundation. Guidelines on colon cancer screening Can J Gastroenteol 2004;18:93-9.

2. Leddin D, Enns R, Hilsden R, Plourde V, Rabeneck L, Sadowski $D$, et al. Canadian Association of Gastroenterology position statement on screening individuals at average risk for developing colorectal cancer, 2010. Can J Gastroenteol 2010;24:705-15. 
3. Sutherland T, Coyle E, Lee WK, Lui B. Diagnosing colorectal polyps and masses: The use of CT colonography. Aust Fam Physician 2011;40:117-20.

4. Van Rijn JC, Reitsma JB, Stoker J, Bossuyt PM, van Deventer SJ, Dekker E. Polyp miss rate determined by tandem colonoscopy: A systematic review. Am J Gastroenterol 2006;101:343-50.

5. Crispin A, Birkner B, Munte A, Nusko G, Mansmann U. Process quality and incidence of acute complications in a series of more than 230,000 outpatient colonoscopies. Endoscopy 2009;41:101825

6. Pickhardt PJ. CT colonography: Time for clinical implementation. Nat Rev Clin Oncol 2009;6:187-8.

7. Lefere P, Dachman AH, Gryspeerdt S. Computer tomographic colonography: Clinical value. Abdom Imaging 2007;32:541-51.

8. Canadian Cancer Society's Steering Committee. Canadian Cancer Statistics 2011. Toronto, ON: Canadian Cancer Society, 2011.

9. Fenlon HM, Nunes DP, Schroy PC, Barish MA, Clarke PD, Ferrucci JT. A comparison of virtual and conventional colonoscopy for the detection of colorectal polyps. N Engl J Med 1999;341:1496-1502.

10. Yee J, Akerkar GA, Hung RK, Steinauer-Gebauer AM. Wall SD, McQuaid KR. Colorectal neoplasia: Performance characteristics of CT colonography for detection in 300 patients. Radiology 2001;219:685-92

11. Pickhardt P, Choi R, Hwang I, Butler JA, Puckett ML, Hildebrandt HA, et al. Computed tomographic virtual colonoscopy to screen for colorectal neoplasia in asymptomatic adults. N Engl J Med 2003;349:2191-200.

12. Mulhall B, Veerappan GR, Jackson JL. Meta-analysis: computed tomographic colonography. Ann Int Med 2005;142:635-50.

13. Halligan S, Altman DG, Taylor SA, Mallett S, Deeks JJ, Bartram C, et al. CT colonography in the detection of colorectal polyps and cancer: systematic review, meta-analysis, and proposed minimum data set for study level reporting. Radiology 2005;237:893-904.

14. Rosman A, Korsten M. Meta-analysis comparing CT colonography, air contrast barium enema, and colonoscopy. Am J Med 2007;120:203-10.

15. Chaparro M, Gisbert J, del Campo L, Cantero J. Accuracy of computed tomographic colonography for the detection of polyps and colorectal tumors: A systematic review and meta-analysis. Digestion 2009;80:1-17.

16. de Haan MC, van Gelder RE, Graser A, Bipat S, Stoker J. Diagnostic value of CT-colonography as compared to colonoscopy in an asymptomatic screening population: a meta-analysis. Eur Radiol 2011;21:1747:63

17. Walleser S, Griffths A, Lord S, Howard K, Solomon M, Gebski $\mathrm{V}$. What is the value of computered tomography colonography in patients screening positive for fecal occult blood? A systematic review and economic analysis. Clin Gastroenterol Hepatol 2007;5:1439-46.

18. Ko C, Dominitz J. Complications of colonoscopy: Magnitude and management. Gastrointest Endosc Clin N Am 2010;20:659-71.

19. Brenner D, Georgsson M. Mass screening with CT colonography: should the radiation exposure be of concern? Gastroenterology 2005;129:328-337

20. Berrington de Gonzalez A, Kim K, Knudsen A, LandsdorpVogelaar L, Rutter CM, Smith-Bindman R, et al. Radiationrelated cancer risks from $\mathrm{CT}$ colonography screening: A risk benefit analysis. Am J Roentgenol 2011;196:816-23.

21. Lansdorp-Vogelaar I, Knudsen A, Brenner H. Cost-effectiveness of colorectal cancer screening. Epidemiol Rev 2011;11:88-100.

22. Behrens C, Stevenson G, Eddy R, Pearson D, Hayashi A, Audet $\mathrm{L}$, et al. The benefit of computed tomographic colonography in reducing a long colonoscopy waiting list. Can Assoc Radiol J 2010;61:33-40.

23. Pickhardt P, Hassan C, Laghi A, Zullo A, Kim D, Morini S. Costeffectiveness of colorectal cancer screening with computed tomography colonography: The impact of not reporting diminutive lesions. Cancer 2007;109:2213-21.

24. Heitman S, Manns B, Hilsden R, Fong A, Dean S, Romagnuolo J. Cost-effectiveness of computerized tomographic colonography versus colonoscopy for colorectal cancer screening. Can Med Assoc J 2005;173:877-81.

25. Heitman S, Au F, Manns B, Pattison P, Hilsden R. Nonmedical costs of colorectal cancer screening using CT colonography. J Am Coll Radiol 2010;7:943-8.

26. Berland L. Incidental extracolonic findings on CT colonography: The impending deluge and its implications. J Am Coll Radiol 2009;6:14-20.

27. Xiong T, McEvoy K, Morton D, Halligan S, Lilford R. Resources and costs associated with incidental extracolonic findings from CT colonography: A study in a symptomatic population. Br J Radiol 2006;79:948-61.

28. Chartier L, Arthurs E, Sewitch M. Patient satisfaction with colonoscopy: A literature review and pilot study. Can J Gastroenterol 2009;23:203-9.

29. Shah H, Paszat L, Saskin R, Stukel T, Rabeneck L. Factors associated with incomplete colonoscopy: A population-based study. Gastroenterology 2007;132:2297-303.

30. Canadian Partnership Against Cancer. Quality Determinants for Colorectal Cancer Screening in Canada. Toronto, ON: Canadian Partnership Against Cancer, 2009.

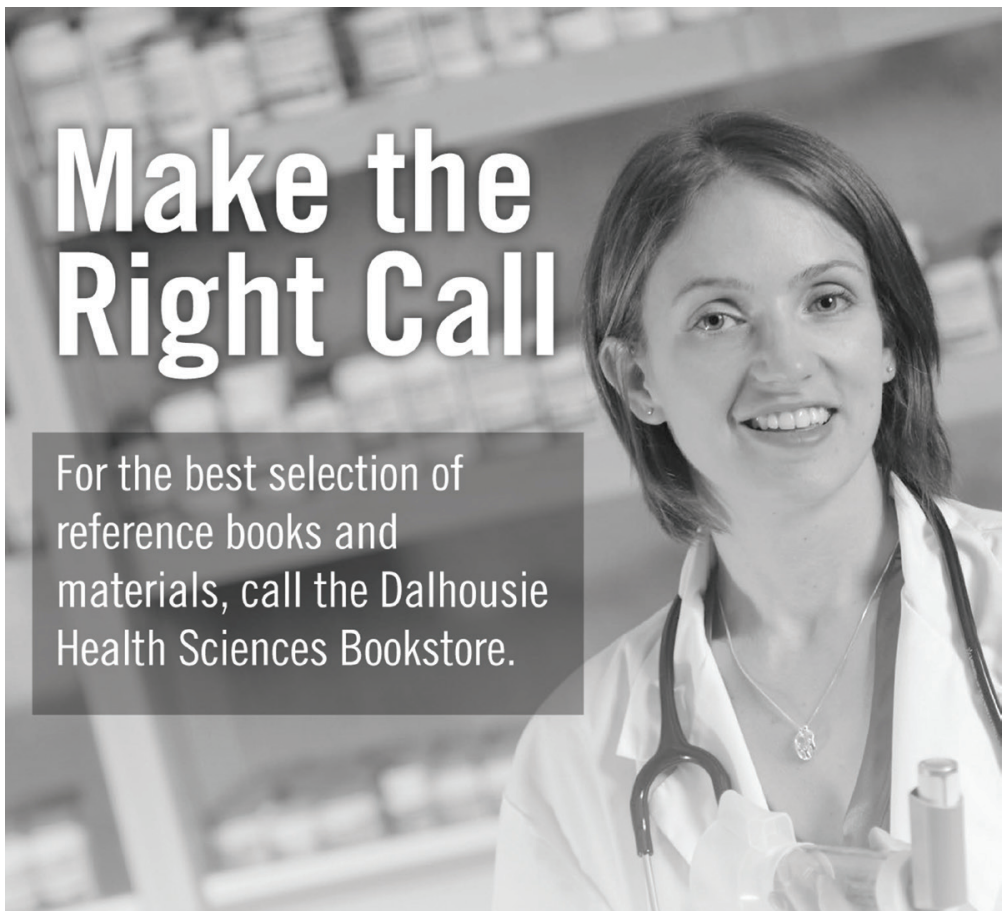

From school to practice, we're always on call for you. Come by or visit our website today.
Health Sciences Bookstore Dentistry Building 5981 University Avenue 902.494 .3020

Toll free: 1-855-258-5988

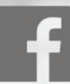

Dalhousie Health Sciences Bookstore VISIT OUR BLOG blogs.dal.ca/hsbookstore
DALHOUSIE UNIVERSITY BOOKSTORES

www.dal.ca/hsbookstore 